\title{
Decentralized Robust Tracking Control for Uncertain Robots
}

\author{
Zongying Shi, Yisheng Zhong and Wenli Xu \\ Tsinghua University \\ P.R. China
}

\section{Introduction}

Theoretically, the motion of rigid robots can be described by a set of nonlinear differential equations or nonlinear differential algebraic equations. In practice, however, because of the model uncertainties such as parameter uncertainty (including unknown or varying payload), unmodelled joint friction, backlash and unknown external disturbances, it is almost impossible to obtain an exact mathematical model of a practical robot system. A lot of work has been done on the motion control of robots with uncertain dynamics (Sage, 1999), and systematic analysis and design methods have been proposed, such as Lyapunov-based algorithms (Spong, 1987 \& 1992; Kelly, 1994; Qu, 1994; Liu \& Goldenberg, 1996 \& 1997), passivity-based algorithms (Canudas et al., 1996) , robust adaptive algorithms (Slotine \& Li, 1987), and so on. Most of these approaches, however, use the upper bound of perturbation to synthesize control laws, so may lead to conservative results. In addition, these approaches are based on a centralized control structure which requires complicated hardware configuration and tedious computation, therefore their practical application is limited.

Decentralized control structure is adopted by the majority of modern robots in favor of its computation simplicity and low-cost hardware set-up (Liu, 1997). For robots, decentralized control means that each joint has its own independent controller which uses only local state feedback. Several decentralized robust tracking approaches have been proposed, such as PD control , PD control plus nonlinear compensation (Liu, 1997 \& 1999; Kelly \& Salgado, 1994; Tang \& Guerrero, 1998), robust adaptive control (Fu, 1992) and Riccati equation based control (Wang \& Weng, 1999). PD controller is the most classical and simplest decentralized controller which results in local stability under sufficient large proportional and differential gains, and the additional nonlinear compensation may $\Phi$ improve the performance of tracking errors from local convergence to global convergence.

$\mathbb{N}$ Riccati equation based approaches may lead to a simple controller, but the involvement of 을 uncertain bound in control laws may lead to conservative results. These approaches consider the torque resulting from the couplings with robot links as exogenous on disturbances, therefore the tuning of the controller parameters of multiple joint robot D systems is a stiff problem.

This chapter presents a design method of decentralized robust controllers for rigid robots. A feedforward control is first applied and the trajectory tracking problem is reformed into a stabilizing problem of a dynamic error system. Then the method, proposed to deal with robust control problem for single-input single-output (SISO) 
plants (Zhong, 1999 \& 2002), is extended to the case of the nonlinear coupled error system which is a multi-input multi-output (MIMO) system. By this method, a robust controller for each joint error subsystem is designed in two steps: first, a nominal controller is designed for the nominal plant to get desired tracking, then, a robust compensator is added to restrain the influence of perturbation that is the difference of the real plant from the nominal plant. Analytical proofs and simulation results show that robust stability and robust tracking property can be achieved in the presence of parameter uncertainty and friction. A novel feature of the presented method is that the parameters of the designed controller can be tuned on-line mono-directionally (more precisely, in the way of increasing a parameter monotonously), and both the format and the bound of uncertainties are not needed to be known when we perform the parameter tuning. This feature greatly facilitates the application of the method to real cases where it is not easy to get the information on the format or the bound of uncertainties involved.

This chapter is organized as follows. Section 1 gives the introduction. Section 2 gives the problem statements. The robust controller design method is shown in Section 3. The stability and robust tracking properties of the closed-loop system are proven in Section 4 . Section 5 gives simulation results on a 2 DOF (degrees of freedom) robot. Section 6 states the conclusions.

\section{Notations}

$\lambda_{\max }(\boldsymbol{B}), \lambda_{\min }(\boldsymbol{B})$ : the maximum and minimum eigenvalues, respectively, of a symmetric positive definite matrix $\boldsymbol{B}$.

$\|x\|=\sqrt{x^{T} x}, x \in \Re^{n}$.

$\|\boldsymbol{A}\|=\sqrt{\lambda_{\max }\left(\boldsymbol{A}^{\mathrm{T}} \boldsymbol{A}\right)}, \boldsymbol{A} \in \mathfrak{R}^{n \times n}$.

\section{Problem statements}

The dynamical model of a rigid robot with $n$ DOFs can be described by its Euler-Lagrange equation

$$
\boldsymbol{M}(\boldsymbol{\theta}) \ddot{\boldsymbol{\theta}}+\boldsymbol{C}(\boldsymbol{\theta}, \dot{\boldsymbol{\theta}}) \dot{\boldsymbol{\theta}}+\boldsymbol{g}(\boldsymbol{\theta})=\boldsymbol{\tau}
$$

where $\boldsymbol{\theta} \in \mathfrak{R}^{n}$ is the vector of angular joint displacements, $\boldsymbol{\tau} \in \mathfrak{R}^{n}$ is the vector of applied joint torques, $\boldsymbol{\tau}_{d} \in \mathfrak{R}^{n}$ is the vector of disturbance torques, $\boldsymbol{M}(\boldsymbol{\theta}) \in \mathfrak{R}^{n \times n}$ is the inertia matrix, $\boldsymbol{C}(\boldsymbol{\theta}, \dot{\boldsymbol{\theta}}) \dot{\boldsymbol{\theta}} \in \mathfrak{R}^{n}$ is the vector of Coriolis and centripetal torques, and $\boldsymbol{g}(\boldsymbol{\theta})$ is the vector of gravitational torques.

In order to incorporate parameter uncertainties into the model (1), the matrices $\boldsymbol{M}(\boldsymbol{\theta})$ and $\boldsymbol{C}(\boldsymbol{\theta}, \dot{\boldsymbol{\theta}})$, and the vector $\boldsymbol{g}(\boldsymbol{\theta})$ are split up into a nominal part (indicated by the subscript zero) and an uncertain part

$$
\left(\boldsymbol{M}_{0}(\boldsymbol{\theta})+\Delta \boldsymbol{M}\right) \ddot{\boldsymbol{\theta}}+\left(\boldsymbol{C}_{0}(\boldsymbol{\theta}, \dot{\boldsymbol{\theta}})+\Delta \boldsymbol{C}\right) \dot{\boldsymbol{\theta}}+\boldsymbol{g}_{0}(\boldsymbol{\theta})+\Delta \boldsymbol{g}=\boldsymbol{\tau}
$$

For the description (1) and (2), the following properties hold which can facilitate control system design and analysis.

Property 1 (Liu, 1997) If all the joints under consideration are revolute, the inertia matrix $\boldsymbol{M}(\boldsymbol{\theta})$ and its inverse matrix $\boldsymbol{M}^{-1}(\boldsymbol{\theta})$ are positive definite, and 


$$
\lambda_{\min }(\boldsymbol{M}) \boldsymbol{I} \leq \boldsymbol{M}(\boldsymbol{\theta}) \leq \lambda_{\max }(\boldsymbol{M}) \boldsymbol{I}
$$

Property 2 For any bounded $\boldsymbol{\theta}_{d}, \dot{\boldsymbol{\theta}}_{d}$ and bounded parameter perturbations, we have

(1) $\left\|\boldsymbol{M}\left(\boldsymbol{\theta}_{d}\right)-\boldsymbol{M}_{0}\left(\boldsymbol{\theta}_{d}\right)\right\| \leq k_{M}$

(2) $\left\|\boldsymbol{C}\left(\boldsymbol{\theta}_{d}, \dot{\boldsymbol{\theta}}_{d}\right)-\boldsymbol{C}_{0}\left(\boldsymbol{\theta}_{d}, \dot{\boldsymbol{\theta}}_{d}\right)\right\| \leq k_{C}$

(3) $\left\|\boldsymbol{g}\left(\boldsymbol{\theta}_{d}\right)-\boldsymbol{g}_{0}\left(\boldsymbol{\theta}_{d}\right)\right\| \leq k_{g}$

where $k_{M}, k_{C}$ and $k_{g}$ are positive constants.

Property 3 (Kelly, 1997) There exist positive constants $k_{M 1}, k_{C 1}, k_{C 2}$ and $k_{g 1}$ such that for all $\boldsymbol{x}, \boldsymbol{y}, \boldsymbol{z}, \boldsymbol{v}, \boldsymbol{w} \in \mathfrak{R}^{n}$, we have

(1) $\|\boldsymbol{M}(\boldsymbol{x}) \boldsymbol{z}-\boldsymbol{M}(\boldsymbol{y}) \boldsymbol{z}\| \leq k_{M 1}\|\boldsymbol{x}-\boldsymbol{y}\|\|z\|$

(2) $\|\boldsymbol{C}(\boldsymbol{x}, \boldsymbol{z}) \boldsymbol{w}-\boldsymbol{C}(\boldsymbol{y}, \boldsymbol{v}) \boldsymbol{w}\| \leq k_{C 1}\|\boldsymbol{z}-\boldsymbol{v}\|\|\boldsymbol{w}\|+k_{C 2}\|\boldsymbol{z}\|\|\boldsymbol{x}-\boldsymbol{y}\|\|\boldsymbol{w}\|$

(3) $\|\boldsymbol{C}(\boldsymbol{x}, \boldsymbol{y}) \boldsymbol{z}\| \leq k_{C 1}\|\boldsymbol{y}\|\|\boldsymbol{z}\|$

(4) $\|\boldsymbol{g}(\boldsymbol{x})-\boldsymbol{g}(\boldsymbol{y})\| \leq k_{g 1}\|\boldsymbol{x}-\boldsymbol{y}\|$

Now, we are in position to formulate the design problem of controller. Consider a control law which consists of a feedforward controller for the nominal system along the desired joint trajectory computed off-line and a robust controller for the real system, given by

$$
\boldsymbol{\tau}=\boldsymbol{u}+\boldsymbol{M}_{0}\left(\boldsymbol{\theta}_{d}\right) \ddot{\boldsymbol{\theta}}_{d}+\boldsymbol{C}_{0}\left(\boldsymbol{\theta}_{d}, \dot{\boldsymbol{\theta}}_{d}\right) \dot{\boldsymbol{\theta}}_{d}+\boldsymbol{g}_{0}\left(\boldsymbol{\theta}_{d}\right)
$$

where subscript zero indicates the corresponding nominal values, $\boldsymbol{\theta}_{d}, \dot{\boldsymbol{\theta}}_{d}$ and $\ddot{\boldsymbol{\theta}}_{d}$ denote the desired joint trajectory and its first and second derivatives respectively, and $\boldsymbol{u}$ is the output of robust controller to be designed.

Substituting the control law (3) into (1) yields the following error dynamics equation

$$
\ddot{\boldsymbol{e}}+\boldsymbol{H}(\boldsymbol{\theta}) \boldsymbol{C}(\boldsymbol{\theta}, \dot{\boldsymbol{\theta}}) \dot{\boldsymbol{e}}+\boldsymbol{H}(\boldsymbol{\theta}) \boldsymbol{\Delta}=\boldsymbol{H}(\boldsymbol{\theta}) \boldsymbol{u}
$$

where

$$
\begin{gathered}
\boldsymbol{e}=\boldsymbol{\theta}-\boldsymbol{\theta}_{d} \\
\boldsymbol{H}(\boldsymbol{\theta})=\boldsymbol{M}^{-1}(\boldsymbol{\theta}) \\
\boldsymbol{\Delta}=\left[\boldsymbol{M}(\boldsymbol{\theta})-\boldsymbol{M}_{0}\left(\boldsymbol{\theta}_{d}\right)\right] \ddot{\boldsymbol{\theta}}_{d}+\left[\boldsymbol{C}(\boldsymbol{\theta}, \dot{\boldsymbol{\theta}})-\boldsymbol{C}_{0}\left(\boldsymbol{\theta}_{d}, \dot{\boldsymbol{\theta}}_{d}\right)\right] \dot{\boldsymbol{\theta}}_{d}+\left[\boldsymbol{g}(\boldsymbol{\theta})-\boldsymbol{g}_{0}\left(\boldsymbol{\theta}_{d}\right)\right]
\end{gathered}
$$

In a practical robot system, each joint is generally driven by an independent actuator, so each joint is treated as a subsystem, whose error dynamics equation can be obtained from (4) and (5)

$$
\ddot{e}_{i}+\sum_{j=1}^{n} \sum_{k=1}^{n} H_{i k}(\boldsymbol{\theta}) C_{k j}(\boldsymbol{\theta}, \dot{\boldsymbol{\theta}}) \dot{e}_{j}+\sum_{j=1}^{n} H_{i j}(\boldsymbol{\theta}) \Delta_{j}(\boldsymbol{\theta})=\sum_{j=1}^{n} H_{i j}(\boldsymbol{\theta}) u_{j}
$$

where $\ddot{e}_{i}$ is the $i$ th element of vector $\ddot{\boldsymbol{e}}, \dot{e}_{j}, u_{j}$ and $\Delta_{j}$ are the $j$ th elements of the vectors $\dot{\boldsymbol{e}}, \boldsymbol{u}$ and $\boldsymbol{\Delta}$ respectively. $H_{i j}(\boldsymbol{\theta})$ and $C_{i j}(\boldsymbol{\theta}, \dot{\boldsymbol{\theta}})$ are the $(i, j)$ elements of the matrices $\boldsymbol{H}(\boldsymbol{\theta})$ and $\boldsymbol{C}(\boldsymbol{\theta}, \dot{\boldsymbol{\theta}})$ respectively.

The remaining part of this paper is to show the design method of a robust controller $u_{i}$ for $i$ th joint subsystem $(i=1, \cdots, n)$ so that the robot tracks the desired joint trajectory $\boldsymbol{\theta}_{d}$ with a specified performance under the condition that $\boldsymbol{\theta}_{d}$ is twice differentiable. 


\section{Robust controller design}

The robust controller for each joint error subsystem is designed by applying the signalcompensation-based (SCB) robust control idea (Zhong, 1999 \& 2002) in two steps: Firstly, an artificial nominal model is introduced and a corresponding nominal controller is designed to achieve desired tracking properties for the nominal closed-loop system. Then, the influence of the difference between the real robot system and its nominal model is regarded as an equivalent disturbance and a robust compensator is designed to restrain the influence of uncertainties and the nonlinear couplings with robot links.

\subsection{Nominal controller design}

An artificial nominal plant is introduced for the $i$ th joint error subsystem

$$
\ddot{e}_{i}=b_{0} u_{i}
$$

where $b_{0}$ is a positive constant such that $b_{0} \geq \lambda_{\max }(\boldsymbol{H})$. Then the nominal plant can be described in frequency domain by

$$
e_{i}=G_{i}(s) u_{i}
$$

where

$$
G_{i}(s)=\frac{b_{0}}{D_{p i}(s)}, \quad D_{p i}(s)=s^{2}
$$

Here we call the nominal plant (7) an artificial one because it might by no means be a model of the real plant for any possible practical situation. Note that the nominal plant (7) is different from that one obtained from (4) by setting $\Delta \boldsymbol{M}=\boldsymbol{0}, \Delta \boldsymbol{C}=\boldsymbol{0}$, and $\Delta \boldsymbol{g}=\boldsymbol{0}$ respectively. Construct the nominal controller, which is a linear time-invariant output dynamical feedback controller, as

$$
u_{0 i}=\frac{N_{y i}(s)}{D_{u i}(s)} e_{i}
$$

where $N_{y i}(s)$ and $D_{u i}(s)$ are polynomials of degree 1 determined by the following equation

$$
D_{m i}(s) L_{i}(s)=D_{p i}(s) D_{u i}(s)-b_{0} N_{y i}(s)
$$

where $D_{m i}(s)$ and $L_{i}(s)$ are Hurwitz monic polynomials of degrees 2 and 1 respectively. Then the closed-loop system consisting of the nominal plant (7) and the nominal controller (9) has a characteristic polynomial $D_{m i}(s) L_{i}(s)$. The existence of the polynomials $N_{y i}(s)$ and $D_{u i}(s)$ satisfying $(10)$ is evident.

Define

$$
D_{u i}(s)=s+d_{1 i}
$$




$$
\begin{gathered}
N_{y i}(s)=r_{0 i} s+r_{1 i} \\
D_{m i}(s)=s^{2}+\beta_{1 i} s+\beta_{2 i} \\
L_{i}(s)=s+\alpha_{1 i}
\end{gathered}
$$

where $D_{m i}(s)$, dominating the performance of $D_{m i}(s) L_{i}(s)$ under the condition $\alpha_{1 i}>>\beta_{1 i}$, is a characteristic polynomial of a 2 degree system which specifies the desired dynamic tracking performance for the $i$ th joint, and $d_{1 i}, r_{0 i}$ and $r_{1 i}$ can be expressed as functions of $\beta_{1 i}, \beta_{2 i}$ and $\alpha_{1 i}$, respectively

$$
\left\{\begin{array}{l}
d_{1 i}=\alpha_{1 i}+\beta_{1 i}>0 \\
r_{0 i}=-\left(\alpha_{1 i} \beta_{1 i}+\beta_{2 i}\right) / b_{0}<0 \\
r_{1 i}=-\alpha_{1 i} \beta_{2 i} / b_{0}<0
\end{array}\right.
$$

\subsection{Robust compensator design}

For the $i$ th joint error subsystem, regarding the effect of the difference between the real robot system and the nominal one as that of an external disturbance, we have

$$
D_{p i}(s) e_{i}=b_{0} u_{i}+q_{i}
$$

where $q_{i}$ is called the equivalent disturbance and given by

$$
q_{i}=\sum_{j=1}^{n} H_{i j}(\boldsymbol{\theta}) u_{j}-b_{0} u_{i}-\sum_{j=1}^{n} \sum_{k=1}^{n} H_{i k}(\boldsymbol{\theta}) C_{k j}(\boldsymbol{\theta}, \dot{\boldsymbol{\theta}}) \dot{e}_{j}-\sum_{j=1}^{n} H_{i j}(\boldsymbol{\theta}) \Delta_{j}
$$

Define the real control input to the $i$ th joint as

$$
u_{i}=u_{0 i}+v_{i}
$$

where $v_{i}$ is the output of the robust compensator to be designed.

To eliminate the influence of $q_{i}$ on the close-loop control system, we set

$$
v_{i}=-F_{i}(s) \frac{q_{i}}{b_{0}}=-F_{i}(s) \frac{1}{b_{0}}\left[D_{p i}(s) e_{i}-b_{0} u_{i}\right]
$$

where $F_{i}(s)=\frac{f_{i}}{s\left(p_{i} s+1\right)+f_{i}}$ is called robust filter with parameters $p_{i}>0, f_{i}>0$.

Combining (10), (13), and (14) yields

$$
v_{i}=-\frac{F(s)}{1-F(s)} \cdot \frac{D_{m i}(s) L_{i}(s)}{b_{0} D_{u i}} e_{i}
$$

and consequently

$$
u_{i}=\left[\frac{r_{0 i} s+r_{1 i}}{s+d_{1 i}}-\frac{f_{i}\left(s^{2}+\beta_{1 i} s+\beta_{2 i}\right)\left(s+\alpha_{1 i}\right)}{b_{0} s\left(p_{i} s+1\right)\left(s+d_{1 i}\right)}\right] e_{i}
$$


which is a linear time-invariant controller using only position feedback, which can avoid the noise problem caused by speed measurement. Fig. 1 shows the configuration of the whole control system with decentralized robust controllers. Each controller appears in a two-loop form: the nominal control loop and the robust control loop. Only when the perturbation from the nominal case appears, the robust control loop is turned on. This feature is very similar to that of an adaptive controller, but the complexity of this controller is almost equivalent to that of a PID controller, therefore its realization is much easier than that of an adaptive controller.

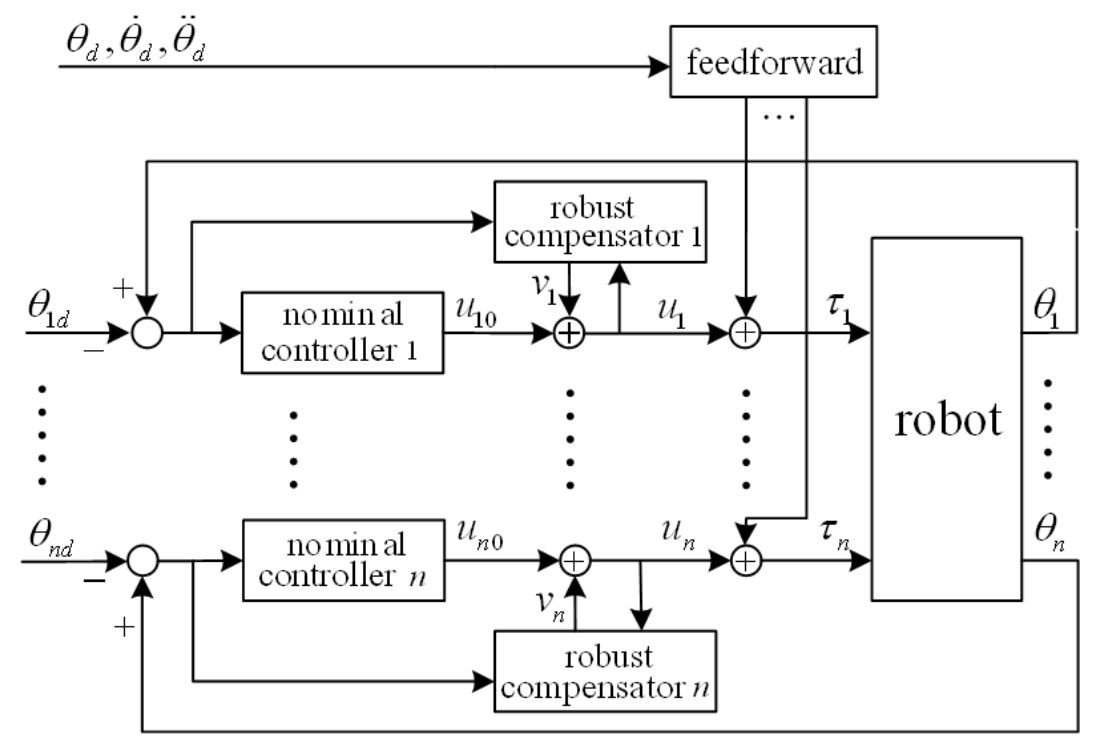

Fig. 1. Decentralized robust control system of $n$ DOF robot.

\section{Control performance analysis and parameter on-line tuning}

\subsection{Control performance analysis}

Firstly we give the state equations of the closed-loop system, then construct a Lyapunov function to analyze the system stability and robust properties.

Let $\boldsymbol{X}_{i}=\left[\begin{array}{lllll}e_{i} & \dot{e}_{i} & u_{0 i} & v_{0 i} & \tilde{v}_{i}\end{array}\right]^{T}$, where $e_{i}, \dot{e}_{i}, u_{0 i}, v_{0 i}$ and $\tilde{v}_{i}$ are the $i$ th elements of vectors $\boldsymbol{e}, \dot{\boldsymbol{e}}, \boldsymbol{u}_{0}, \boldsymbol{v}_{0}$ and $\tilde{\boldsymbol{v}}$ respectively, and $\tilde{v}_{i}=-v_{i} / \sqrt{f_{i}}$. Combining (9), (11) and (14) results in the state equation of the $i$ th joint error subsystem:

$$
\begin{gathered}
\dot{\boldsymbol{X}}_{i}=\boldsymbol{A}_{i} \boldsymbol{X}_{i}+\boldsymbol{B}_{q i} q_{i} \\
\boldsymbol{A}_{i}=\left[\begin{array}{ccccc}
0 & 1 & 0 & 0 & 0 \\
0 & 0 & b_{0} & 0 & -b_{0} \sqrt{f_{i}} \\
r_{11} & r_{0 i} & -d_{1 i} & 0 & 0 \\
0 & 0 & 0 & \lambda_{1 i} & 0 \\
0 & 0 & 0 & 1 / p_{i} & \lambda_{2 i}
\end{array}\right], \quad B_{q i}=\left[\begin{array}{c}
0 \\
1 \\
0 \\
\sqrt{f_{i}} / b_{0} \\
0
\end{array}\right]
\end{gathered}
$$


where $\lambda_{1 i}$ and $\lambda_{2 i}$ are the poles of the robust filter or the roots of the polynomial $s\left(p_{i} s+1\right)+f_{i}$, that is, $\lambda_{i, 2 i}=\frac{1}{2}\left(-\frac{1}{p_{i}} \pm \sqrt{\frac{1}{p_{i}^{2}}-\frac{4 f_{i}}{p_{i}}}\right)$.

The characteristic polynomial of matrix $\boldsymbol{A}_{i}$ is

$$
\Lambda_{i}(s)=D_{m i}(s) L_{i}(s) \frac{1}{p_{i}}\left(s\left(p_{i} s+1\right)+f_{i}\right)
$$

which is a Hurwitz polynomial. Therefore matrix $\boldsymbol{A}_{i}$ is stable and there exists a positive definite matrix $\boldsymbol{P}_{i}$ which is the solution of the following Lyapunov equation.

$$
\boldsymbol{P}_{i} \boldsymbol{A}_{i}+\boldsymbol{A}_{i}^{T} \boldsymbol{P}_{i}=-\boldsymbol{I}
$$

\section{Assumption}
A) $\frac{1}{p_{i}}>>f_{i} \geq 1$.
B) $f_{i}=f_{j}, i \neq j, \boldsymbol{f}=\operatorname{diag}\left\{f_{1}, \cdots, f_{n}\right\}=f_{i} \boldsymbol{I}_{n \times n}$.
C) $\varepsilon_{\Delta}$ is a positive constant such that $\varepsilon_{\Delta} \leq b_{0}^{-1} \lambda_{\min }\left(\boldsymbol{M}^{-1}\right)$.

Remark 1 Under Assumption A), we have that $1 \gg p_{i} f_{i}$. Since $\boldsymbol{M}(\boldsymbol{\theta})$ (hence $\boldsymbol{M}^{-1}(\boldsymbol{\theta})$ ) is a positive definite matrix, the constant $\varepsilon_{\Delta}$ satisfying Assumption C) always exists.

Lemma 1 Define $\boldsymbol{\psi}_{i}=\boldsymbol{P}_{i} \boldsymbol{B}_{q i}$ and $\frac{1}{p_{i}}=\mu f_{i}$, where $\mu>>1$, then vector $\boldsymbol{\psi}_{i}$ can be represented in the following form

$$
\boldsymbol{\psi}_{i}=\frac{1}{b_{0 i} \sqrt{f_{i}}}\left[\frac{\gamma_{1 i}}{\sqrt{f_{i}}} \frac{\gamma_{2 i}}{\sqrt{f_{i}}} \frac{\gamma_{3 i}}{\sqrt{f_{i}}} \quad 1+\frac{\gamma_{4 i}}{\mu} \frac{\gamma_{5 i}}{\mu}\right]^{T}
$$

where $\gamma_{j i}(j=1, \cdots, 5)$ are bounded by constants independent of $f_{i}$.

Proof: See the Appendix.

Lemma 2 Define the equivalent disturbance $q_{i}$ of $i$ th joint error subsystem as (12), then the total equivalent disturbance $\boldsymbol{q}$ of system (4) can be expressed as

$$
\boldsymbol{q}=\tilde{\boldsymbol{q}}+\left(\boldsymbol{M}^{-1}-b_{0} \boldsymbol{I}\right) \boldsymbol{v}
$$

where $\boldsymbol{q}=\left[\begin{array}{lll}q_{1} & \cdots & q_{n}\end{array}\right]^{T}, \tilde{\boldsymbol{q}}=-\boldsymbol{M}^{-1} \boldsymbol{C} \dot{\boldsymbol{e}}-\boldsymbol{M}^{-1} \boldsymbol{\Delta}+\left(\boldsymbol{M}^{-1}-b_{0} \boldsymbol{I}\right) \boldsymbol{u}_{0}$, and there exist positive constants $k_{q 1}, k_{q 2}, \cdots, k_{q 5}$ such that

$$
\|\tilde{\boldsymbol{q}}\| \leq k_{q 1}+k_{q 2}\|\boldsymbol{e}\|+k_{q 3}\|\dot{\boldsymbol{e}}\|+k_{q 4}\|\dot{\boldsymbol{e}}\|^{2}+k_{q 5}\left\|\boldsymbol{u}_{0}\right\|
$$

Proof: See the Appendix.

Lemma 3 Define $\boldsymbol{A} \in \mathfrak{R}^{n \times n}$ for $\bar{\sigma}(\boldsymbol{A})=\|\boldsymbol{A}\|$. Under Assumption C), we have

$$
\bar{\sigma}\left[b_{0}^{-1}\left(\boldsymbol{M}^{-1}-b_{0} \boldsymbol{I}\right)\right] \leq 1-\varepsilon_{\Delta}
$$


Proof: See the Appendix.

Lemma 4 Define $\mu_{0}$ as

$$
\mu_{0}=\frac{k_{q 1}}{b_{0} \sqrt{f_{i}}} \sum_{i=1}^{3} \gamma_{i \max }+\frac{k_{q 1}}{b_{0}}\left[1+\left(\gamma_{4 \max }+\gamma_{5 \max }\right) / \mu\right]
$$

Under assumption A), if $f_{i} \geq f_{c 1}$, where $f_{c 1}=\max _{j=1}^{3}\left(\gamma_{j \max }\right)^{2}, \gamma_{j \max }=\max \left\{0, \max _{i=1}^{n}\left(\gamma_{j i}\right)\right\}$, $j=1, \cdots, 5$ then we have

$$
\mu_{0} \leq \frac{5 k_{q 1}}{b_{0}}
$$

Proof: Omitted.

Theorem 1 Suppose Assumptions A), B) and C) are met. For any given constant $\eta>0$, there exists a bounded $f_{i}$ such that the closed-loop system has the following robust properties:

(1) If both the closed-loop system and the reference model are of zero initial conditions, then the closed-loop system has robust transient property:

$$
\|X\|^{2}<\eta, \forall t \geq t_{0}
$$

(2) If the initial conditions involved are non-zero but bounded, then the closed-loop system has robust asymptotical tracking property; that is, there exists a constant $T \geq t_{0}$ such that

$$
\|X\|^{2}<\eta, \forall t \geq T
$$

Proof: Consider the following Lyapunov function candidate

$$
V=\boldsymbol{X}^{T} \boldsymbol{P} \boldsymbol{X}=\sum_{i=1}^{n} V_{i}, V_{i}=\boldsymbol{X}_{i}^{T} \boldsymbol{P}_{i} \boldsymbol{X}_{i}
$$

where

$$
\boldsymbol{X}=\left[\boldsymbol{X}_{1}^{T}, \boldsymbol{X}_{2}^{T}, \cdots, \boldsymbol{X}_{n}^{T}\right]^{T}, \boldsymbol{P}=\left[\begin{array}{lll}
\boldsymbol{P}_{1} & & \\
& \ddots & \\
& & \boldsymbol{P}_{n}
\end{array}\right]
$$

The derivative of $V$, along the solution of the controlled system (16), is given by

$$
\begin{aligned}
& \dot{V}=\sum_{i=1}^{n} \dot{V}_{i}=\sum_{i=1}^{n}\left(-\boldsymbol{X}_{i}^{T} \boldsymbol{X}_{i}+2 \boldsymbol{X}_{i}^{T} \boldsymbol{P}_{i} \boldsymbol{B}_{q i} q_{i}\right) \\
& \leq-\left(\boldsymbol{e}^{T} \boldsymbol{e}+\dot{\boldsymbol{e}}^{T} \dot{\boldsymbol{e}}+\boldsymbol{u}_{0}^{T} \boldsymbol{u}_{0}+\boldsymbol{v}_{0}^{T} \boldsymbol{v}_{0}+\tilde{\boldsymbol{v}}^{T} \tilde{\boldsymbol{v}}\right)+2\left\{\sum_{i=1}^{n} \frac{1}{f_{i} b_{0}} \gamma_{1 i} e_{i} q_{i}+\sum_{i=1}^{n} \frac{1}{f_{i} b_{0}} \gamma_{2 i} \dot{e}_{i} q_{i}\right. \\
& \left.+\sum_{i=1}^{n} \frac{1}{f_{i} b_{0}} \gamma_{3 i} u_{0 i} q_{i}+\sum_{i=1}^{n} \frac{1}{\sqrt{f_{i}} b_{0}}\left(1+\frac{\gamma_{4 i}}{\mu}\right) v_{0 i} q_{i}+\sum_{i=1}^{n} \frac{\gamma_{5 i}}{\sqrt{f_{i}} b_{0} \mu} \tilde{v}_{i} q_{i}\right\}
\end{aligned}
$$




$$
\begin{aligned}
= & -\left(\boldsymbol{e}^{T} \boldsymbol{e}+\dot{\boldsymbol{e}}^{T} \dot{\boldsymbol{e}}+\boldsymbol{u}_{0}^{T} \boldsymbol{u}_{0}+\boldsymbol{v}_{0}^{T} \boldsymbol{v}_{0}+\tilde{\boldsymbol{v}}^{T} \tilde{\boldsymbol{v}}\right)+2\left[\boldsymbol{e}^{T} \operatorname{diag}\left\{\frac{\gamma_{1 i}}{f_{i} b_{0}}\right\}+\dot{\boldsymbol{e}}^{T} \operatorname{diag}\left\{\frac{\gamma_{2 i}}{f_{i} b_{0}}\right\}\right. \\
& \left.+\boldsymbol{u}_{0}^{T} \operatorname{diag}\left\{\frac{\gamma_{3 i}}{f_{i} b_{0}}\right\}+\boldsymbol{v}_{0}^{T} \operatorname{diag}\left\{\frac{1}{\sqrt{f_{i}} b_{0}}\left(1+\frac{\gamma_{4 i}}{\mu}\right)\right\}+\tilde{\boldsymbol{v}}^{T} \operatorname{diag}\left\{\frac{\gamma_{5 i}}{\sqrt{f_{i}} b_{0} \mu}\right\}\right] \boldsymbol{q}
\end{aligned}
$$

From Lemma 2 and the definition of $\gamma_{j \max }$ in Lemma 4, (22) becomes

$$
\begin{gathered}
\dot{V} \leq-\left(\|\boldsymbol{e}\|^{2}+\|\dot{\boldsymbol{e}}\|^{2}+\left\|\boldsymbol{u}_{0}\right\|^{2}+\left\|\boldsymbol{v}_{0}\right\|^{2}+\|\tilde{\boldsymbol{v}}\|^{2}\right) \\
+2\left\{\left[\frac{1}{f_{i} b_{0}}\left(\gamma_{1 \max }\|\boldsymbol{e}\|+\gamma_{2 \max }\|\dot{\boldsymbol{e}}\|+\gamma_{3 \max }\left\|\boldsymbol{u}_{0}\right\|\right)+\frac{1}{\sqrt{f_{i}} b_{0}}\left(1+\frac{\gamma_{4 \max }}{\mu}\right)\left\|\boldsymbol{v}_{0}\right\|+\frac{\gamma_{5 \max }}{b_{0} \mu}\|\tilde{\boldsymbol{v}}\|\right] \cdot\|\tilde{\boldsymbol{q}}\|\right. \\
+\left[\frac{1}{\sqrt{f_{i}}}\left(\gamma_{1 \max }\|\boldsymbol{e}\|+\gamma_{2 \max }\|\dot{\boldsymbol{e}}\|+\gamma_{3 \max }\left\|\boldsymbol{u}_{0}\right\|\right)\right. \\
\left.\left.+\left(1+\frac{\gamma_{4 \max }}{\mu}\right)\left\|v_{0}\right\|+\frac{\gamma_{5 \max }}{\mu}\|\tilde{\boldsymbol{v}}\|\right] \cdot\left\|b_{0}^{-1}\left(\boldsymbol{M}^{-1}-b_{0} \boldsymbol{I}\right)\right\| \cdot\|\tilde{\boldsymbol{v}}\|\right\}
\end{gathered}
$$

In the view of Lemma 1 and Lemma 3, (23) becomes

$$
\begin{aligned}
& \dot{V} \leq-\left(\|\boldsymbol{e}\|^{2}+\|\dot{\boldsymbol{e}}\|^{2}+\left\|\boldsymbol{u}_{0}\right\|^{2}+\left\|\boldsymbol{v}_{0}\right\|^{2}+\|\tilde{\boldsymbol{v}}\|^{2}\right) \\
& +2\left\{\left[\frac{1}{f_{i} b_{0}}\left(\gamma_{1 \max }\|\boldsymbol{e}\|+\gamma_{2 \max }\|\dot{\boldsymbol{e}}\|+\gamma_{3 \max }\left\|\boldsymbol{u}_{0}\right\|\right)+\frac{1}{\sqrt{f_{i}} b_{0}}\left(1+\frac{\gamma_{4 \max }}{\mu}\right)\left\|\boldsymbol{v}_{0}\right\|\right.\right. \\
& \left.+\frac{\gamma_{5 \max }}{b_{0} \mu}\|\tilde{\boldsymbol{v}}\|\right] \cdot\left[k_{q 1}+k_{q 2}\|\boldsymbol{e}\|+k_{q 3}\|\dot{\boldsymbol{e}}\|+k_{q 4}\|\dot{\boldsymbol{e}}\|^{2}+k_{q 5}\left\|\boldsymbol{u}_{0}\right\|\right] \\
& +\frac{1}{\sqrt{f_{i}}}\left(\gamma_{1 \max }\|\boldsymbol{e}\|+\gamma_{2 \max }\|\dot{\boldsymbol{e}}\|+\gamma_{3 \max }\left\|\boldsymbol{u}_{\boldsymbol{0}}\right\|\right)\|\tilde{\boldsymbol{v}}\| \\
& \left.+\bar{\delta}\left(b_{0}^{-1}\left(\boldsymbol{M}^{-1}-b_{0} \boldsymbol{I}\right)\right)\left[\left(1+\frac{\gamma_{4 \max }}{\mu}\right)\left\|\boldsymbol{v}_{0}\right\|+\frac{\gamma_{5 \max }}{\mu}\|\tilde{\boldsymbol{v}}\|\right] \cdot\|\tilde{\boldsymbol{v}}\|\right\}
\end{aligned}
$$

Define $k_{\text {sum }}=k_{q 1}+k_{q 2}+k_{q 3}+k_{q 5}$ and $\boldsymbol{k}=\left[k_{q 2}, k_{q 3}, k_{q 5}\right]^{T}$, and let $k_{i}$ denote the $i$ th element of the vector $\boldsymbol{k}$. Then (24) is further expressed as

$$
\dot{V} \leq-\mu_{1}\|\boldsymbol{e}\|^{2}-\mu_{2}\|\dot{\boldsymbol{e}}\|^{2}-\mu_{3}\left\|\boldsymbol{u}_{0}\right\|^{2}-\mu_{4}\left\|\boldsymbol{v}_{0}\right\|^{2}-\mu_{5}\|\tilde{\boldsymbol{v}}\|^{2}+\frac{\mu_{0}}{\sqrt{f_{i}}}
$$

where $\mu_{0}$ is defined as (20), 


$$
\begin{gathered}
\mu_{i}= \begin{cases}1-\rho_{i} / \sqrt{f_{i}} & (i=1,3) \\
1-\left(\tilde{\rho}_{i}+\rho_{i}\right) / \sqrt{f_{i}} & (i=2) \\
1-\bar{\delta}\left(b_{0}^{-1}\left(\boldsymbol{M}^{-1}-b_{0} \boldsymbol{I}\right)\right)\left(1+\gamma_{4 \max } / \mu\right)-\rho_{i} / \sqrt{f_{i}} & (i=4) \\
1-\bar{\delta}\left(b_{0}^{-1}\left(\boldsymbol{M}^{-1}-b_{0} \boldsymbol{I}\right)\right)\left[1+\left(\gamma_{4 \max }+2 \gamma_{5 \max }\right) / \mu\right]-\rho_{i} / \sqrt{f_{i}} & (i=5)\end{cases} \\
\rho_{i}= \begin{cases}\frac{1}{\sqrt{f_{i}} b_{0}}\left[\gamma_{i \max } k_{\text {sum }}+k_{i} \sum_{j=1}^{3} \gamma_{j \max }\right]+\frac{k_{i}}{b_{0}}\left[1+\frac{\gamma_{4 \max }+\gamma_{5 \max }}{\mu}\right]+\gamma_{i \max } & (i=1,2,3) \\
b_{0}\left[\frac{\gamma_{4 \max }}{\mu}\right] k_{\text {sum }} & (i=4) \\
\sum_{j=1}^{3} \gamma_{j \max }+\frac{\gamma_{5 \max } k_{\text {sum }}}{b_{0} \mu} & (i=5)\end{cases} \\
\tilde{\rho}_{2}=\frac{2}{\lambda_{b}}\left[\frac{\gamma_{1 \max }}{\sqrt{f_{i}}}\|\boldsymbol{e}\|+\frac{\gamma_{2 \max }}{\sqrt{f_{i}}}\|\dot{\boldsymbol{e}}\|+\frac{\gamma_{3 \max }}{\sqrt{f_{i}}}\left\|\boldsymbol{u}_{0}\right\|+\left(1+\frac{\gamma_{4 \max }}{\mu}\right)\left\|\boldsymbol{v}_{0}\right\|+\frac{\gamma_{5 \max }}{\mu}\|\tilde{\boldsymbol{v}}\|\right] k_{q 4}
\end{gathered}
$$

Define $\boldsymbol{Y}=\left[\boldsymbol{e}^{T}, \dot{\boldsymbol{e}}^{T}, \boldsymbol{u}_{0}^{T}, \boldsymbol{v}_{0}^{T}, \tilde{\boldsymbol{v}}^{T}\right]^{T}=\left[\boldsymbol{Y}_{1}^{T}, \boldsymbol{Y}_{2}^{T}, \boldsymbol{Y}_{3}^{T}, \boldsymbol{Y}_{4}^{T}, \boldsymbol{Y}_{5}^{T}\right]^{T}$, then under Assumption A), we have

$$
\tilde{\rho}_{2} \leq \phi_{1} \sum_{j=1}^{5}\left\|\boldsymbol{Y}_{i}\right\| \leq \sqrt{5} \phi_{1}\|\boldsymbol{Y}\|
$$

where $\phi_{1}=2 k_{q 4} b_{0}^{-1} \max \left\{\gamma_{1 \max }, \gamma_{2 \max }, \gamma_{3 \max }, 2\right\}$, is a bounded constant independent of $f_{i}$.

In the following, the conditions are analyzed that ensure $\mu_{i}>0, i=1,2, \cdots, 5$.

Let $\pi_{\min }$ be a positive constant so that $\pi_{\min }<\min \left\{\varepsilon_{\Delta}, 1\right\}$, and let $\bar{\rho}_{i}$ denote the values of $\rho_{i}$ when $f_{i}=1$ and $\mu=\gamma_{4 \max }+2 \gamma_{5 \max }$. Then under Assumption A) and the condition that $\mu \geq \gamma_{4 \max }+2 \gamma_{5 \max }$, we have $\rho_{i} \leq \bar{\rho}_{i}$.

If the following inequalities hold

$$
\begin{gathered}
f_{i} \geq f_{c 2}, \quad \mu \geq \gamma_{4 \max }+2 \gamma_{5 \max } \\
\|Y\| \leq \frac{1}{\sqrt{5} \phi_{1}}\left[\left(1-\pi_{\min }\right) \sqrt{f_{i}}-\bar{\rho}_{2}\right]
\end{gathered}
$$

where

$$
f_{c 2}=\max \left\{\max _{i=1,3}\left(\frac{\bar{\rho}_{i}}{1-\pi_{\min }}\right)^{2}, \max _{i=4,5}\left(\frac{\bar{\rho}_{i}}{\varepsilon_{\Delta}-\pi_{\min }}\right)^{2}\right\}
$$


then by (26) and from Lemma 3, we have

$$
\mu_{i} \geq \pi_{\min }, i=1,2, \cdots, 5
$$

Note that (31) defines the attractive region.

If (33) holds and $f_{i} \geq f_{c 1}$, from Lemma 4 , one sees that (25) can be rewritten as

$$
\dot{V} \leq-\pi_{\min }\|\boldsymbol{Y}\|^{2}+\frac{5 k_{q 1}}{\sqrt{f_{i}} b_{0}}
$$

Since

$$
\|\boldsymbol{Y}\|^{2}=\|\boldsymbol{X}\|^{2} \geq \frac{\boldsymbol{X}^{T} \boldsymbol{P} \boldsymbol{X}}{\lambda_{\max }(\boldsymbol{P})}=\frac{V}{\lambda_{\max }(\boldsymbol{P})}
$$

we obtain

$$
\dot{V} \leq-\zeta_{s} V+\varepsilon_{s}
$$

and

$$
V(t) \leq V\left(t_{0}\right) e^{-\zeta_{s}\left(t-t_{0}\right)}+\frac{\varepsilon_{s}}{\zeta_{s}}\left(1-e^{-\zeta_{s}\left(t-t_{0}\right)}\right)
$$

where $\zeta_{s}=\frac{\pi_{\min }}{\lambda_{\max }(\boldsymbol{P})}, \varepsilon_{s}=\frac{5 k_{q 1}}{\sqrt{f_{i}} b_{0}}$.

Let

$$
\bar{V}=\max \left\{V\left(t_{0}\right), 5 k_{q 1} b_{0}^{-1} \zeta_{s}^{-1}\right\}
$$

Then under Assumption A) and from (35) it is known that $V(t) \leq \bar{V}, \forall t \geq t_{0}$ and $\lim _{t \rightarrow \infty} V(t) \leq \varepsilon_{s} \zeta_{s}^{-1}$. Because

$$
\|\boldsymbol{Y}\|^{2}=\|\boldsymbol{X}\|^{2} \leq \frac{\boldsymbol{X}^{T} \boldsymbol{P} \boldsymbol{X}}{\lambda_{\text {min }}(\boldsymbol{P})}=\frac{V}{\lambda_{\text {min }}(\boldsymbol{P})}
$$

$\boldsymbol{Y}(t)$ (and $\boldsymbol{X}(t)$ ) starting from the attractive region given by (31) can keep staying inside that region, if

$$
\frac{1}{\sqrt{5} \phi_{1}}\left[\left(1-\pi_{\min }\right) \sqrt{f_{i}}-\bar{\rho}_{2}\right] \geq \sqrt{\bar{V} \lambda_{\min }^{-1}(\boldsymbol{P})}
$$

By Assumption A), (38) holds, if

$$
f_{i}>f_{c 3}, f_{c 3}=\frac{1}{\left(1-\pi_{\min }\right)^{2}}\left(\phi_{1} \sqrt{5 \bar{V} \lambda_{\min }^{-1}(\boldsymbol{P})}+\bar{\rho}_{2}\right)^{2}
$$


In addition, from (35) and (37) we know that the state $\boldsymbol{Y}$ convergences to the set given by

$$
\Omega_{s}=\left\{\boldsymbol{Y} \mid\|\boldsymbol{Y}\|^{2} \leq \varepsilon_{s} /\left(\zeta_{s} \lambda_{\min }(\boldsymbol{P})\right)\right\}
$$

at a rate not slower than $\exp \left(-\zeta_{s} t / 2\right)$.

By (37), if

$$
V(t) \leq \lambda_{\min }(\boldsymbol{P}) \eta
$$

then

$$
\|\boldsymbol{X}\|^{2} \leq \eta
$$

When both of the closed-loop system and the reference model are of zero initial conditions, $V\left(t_{0}\right)=0$. In this case, from (37), we have $\|\boldsymbol{X}\|_{t \geq t_{0}}^{2}<\eta$, if

$$
\frac{\varepsilon_{s}}{\zeta_{s}} \leq \eta \lambda_{\min }(\boldsymbol{P})
$$

In the case of non-zero initial states, $V\left(t_{0}\right) \neq 0$. We can obtain $\|\boldsymbol{X}\|_{t \geq T}^{2}<\eta$, if

$$
V\left(t_{0}\right) e^{-\zeta_{s}\left(t-t_{0}\right)}+\frac{\varepsilon_{s}}{\zeta_{s}} \leq \eta \lambda_{\min }(\boldsymbol{P})
$$

If $T \geq t_{0}+\frac{1}{\zeta_{s}} \ln \left(\frac{2 V\left(t_{0}\right)}{\eta \lambda_{\min }(\boldsymbol{P})}\right)$, we have

$$
V\left(t_{0}\right) e^{-\zeta_{s}\left(T-t_{0}\right)} \leq \frac{1}{2} \eta \lambda_{\min }(\boldsymbol{P})
$$

Now to ensure (41) hold, it is required that

$$
\frac{\varepsilon_{s}}{\zeta_{s}} \leq \frac{1}{2} \eta \lambda_{\min }(\boldsymbol{P})
$$

Incorporating (40) and (42) yields

$$
f_{i} \geq f_{c 4}, f_{c 4}=\left(\frac{10 k_{q 1} \lambda_{\max }(\boldsymbol{P})}{\eta b_{0} \pi_{\min } \lambda_{\min }(\boldsymbol{P})}\right)^{2}
$$

From the previous analysis, we see that it is required

$$
f_{i} \geq \max _{j=1}^{4} f_{c j}, \mu \geq \gamma_{4 \max }+2 \gamma_{5 \max }
$$


where $f_{c j}, j=1, \cdots, 4$ are bounded constants and $\gamma_{4 \max }+2 \gamma_{5 \max }$ are bounded by a constant, all independent of $f_{i}$. When the parameters $f_{i}$ and $\mu$ are set such that the above conditions hold, then the robust transient property and robust tracking property can be achieved. So the conclusions of the theorem are proven.

Remark 2 If the friction torque is bounded by $\beta_{1}+\beta_{2}\|\dot{\boldsymbol{\theta}}\|++\beta_{3}\|\dot{\boldsymbol{\theta}}\|^{2}$, where $\beta_{1}, \beta_{2}$, and $\beta_{3}$ are positive constants, then the upper bound of $\tilde{\boldsymbol{q}}$ also can be expressed as the form of (19), therefore the conclusions of Theorem 1 are still available.

\subsection{Parameter on-line tuning}

In practical applications, needing not the tedious analysis and estimation of the uncertainty as in the previous proof, the parameters of robust compensator can be determined by online tuning as follows: 1 ) let $\mu>>1$, and $p_{i}=1 /\left(\mu f_{i}\right)$ (From Lemma 4, Theorem 1, and their proofs, one sees that it is enough to set $\left.\mu \geq \gamma_{4 \max }+2 \gamma_{5 \max }\right) ; 2$ ) construct the controller as (15) and close the control loop; 3 ) tune $f_{i}$ monotonously from some value greater than or equal to 1 until the satisfied tracking performance is achieved.

Because there is only one parameter to be tuned and the tuning is monotonously increasing, the parameter on-line tuning can be performed even easier than that for a traditional PID controller. In addition, the controller tuning of each joint subsystem is independent of each other.

In real cases, however, we should not apply a too large $f_{i}$ to avoid too large control input caused by nonzero initial conditions or measure noise.

For a real system, it is difficult to exactly determine the range of uncertain parameters, so the feature of parameter on-line tuning of this controller possesses its unique superiority.

\section{Numerical example and simulation results}

We apply the method proposed in previous sections to a 2DOFs manipulator (Liu, 1999) to verify the monotonously tuning characteristic and effectiveness of the controller. The manipulator's physical parameters and their real values are shown in Table 1 . The entries of $\boldsymbol{M}(\boldsymbol{\theta}), \boldsymbol{C}(\boldsymbol{\theta}, \dot{\boldsymbol{\theta}})$ and $\boldsymbol{g}(\boldsymbol{\theta})$ are as follows respectively.

$$
M_{11}=J_{11}+2 J_{22} \cos \theta_{2}, M_{12}=M_{21}=J_{21}+J_{22} \cos \theta_{2}, M_{22}=J_{21}
$$

Let $\boldsymbol{h}(\boldsymbol{\theta}, \dot{\boldsymbol{\theta}})=\boldsymbol{C}(\boldsymbol{\theta}, \dot{\boldsymbol{\theta}}) \dot{\boldsymbol{\theta}}$, then we have

$$
\begin{aligned}
& h_{1}=-J_{22}\left(2 \dot{\theta}_{1} \dot{\theta}_{2}+\dot{\theta}_{2}^{2}\right) \sin \theta_{2}, h_{2}=J_{22} \dot{\theta}_{1}^{2} \sin \theta_{2} \\
& g_{1}=J_{12} \cos \theta_{1}+m_{2} g r_{2} \cos \left(\theta_{1}+\theta_{2}\right), g_{2}=m_{2} g r_{2} \cos \left(\theta_{1}+\theta_{2}\right)
\end{aligned}
$$

where $J_{i j}$ are constant parameters given by

$$
\begin{aligned}
& J_{11}=m_{1} l_{c 1}^{2}+m_{2}\left(l_{1}^{2}+l_{c 2}^{2}\right)+I_{1}+I_{2} \\
& J_{12}=g\left(m_{1} l_{c 1}+m_{2} l_{1}\right)
\end{aligned}
$$




$$
J_{21}=m_{2} l_{c 2}^{2}+I_{2}, J_{22}=m_{2} l_{1} l_{c 2}
$$

The nominal values of robot's physical parameters are $\hat{m}_{1}=3.5 \mathrm{~kg}, \hat{m}_{2}=1.7 \mathrm{~kg}, \hat{I}_{1}=0.9 \mathrm{kgm}^{2}$, $\hat{I}_{2}=0.7 \mathrm{kgm}^{2}, \hat{l}_{i}=l_{i}, \hat{l}_{c i}=l_{c i}, i=1,2$. Let the initial tracking error $e=\left[\begin{array}{ll}0.2 & -0.2\end{array}\right]^{T}(\mathrm{rad})$ and $\dot{e}=\left[\begin{array}{ll}-0.25 & 0.2\end{array}\right]^{T}(\mathrm{rad} / \mathrm{s})$.

The reference trajectories are also specified as in (Liu, 1999) for comparison:

$$
\begin{aligned}
& \theta_{1 d}=0.2+2 \sin 2 t \quad(\mathrm{rad}) \\
& \theta_{2 d}=-1.7+1.8 \cos 2 t \quad(\mathrm{rad})
\end{aligned}
$$

The 2 degree reference model is set as follows: the rise-time $t_{r}=0.5 \mathrm{~s}$, the damp coefficient $\xi=1.1$. Thus $D_{m i}(s)=s^{2}+10.1 s+21.1$. Set $\mu=10\left(>\gamma_{4 \max }+2 \gamma_{5 \max }=5\right)$, and the

\begin{tabular}{|l|c|c|c|}
\hline Items & symbol & Link1 & Link2 \\
\hline Link length(m) & $l_{i}$ & 0.5 & 0.25 \\
\hline Distance from joint axis to COM of link(m) & $l_{c i}$ & 0.25 & 0.15 \\
\hline Link mass(kg) & $m_{i}$ & 4.0 & 2.0 \\
\hline Moment of inertia of link $\left(\mathrm{kgm}^{2}\right)$ & $I_{i}$ & 1.0 & 0.8 \\
\hline
\end{tabular}

Table 1. Physical parameters of a 2DOFs manipulator.

value of $f_{i}$ is tuned from 1.0. The tracking errors of uncertain robot with parameter uncertainty corresponding to $f_{i}=10, f_{i}=20$ and $f_{i}=50$ are shown in Fig. 2 and Fig. 3. The results indicate that, along with the gradual increment of $f_{i}$, tracking errors gradually decrease and tracking performance is improved, which validates the monotonously tuning characteristic of the controller.

Fig. $4 \sim$ Fig. 7 show the tracking errors of three control schemes with almost equivalent proportional gain: signal-compensation-based (SCB) control law proposed in this paper, PDplus-nonlinear (PD+NL) control law (Liu, 1999) and PD-plus-nonlinear-plus-adaptive (PD+NL+AD) control law (Liu, 1999). It can be seen that the tracking performance of SCB control is superior to that of PD+NL control and not inferior to that of PD+NL+AD control. The input torque signals are shown in Fig. 8 and Fig. 9, which indicate that, after a very short initial phase, the input torque curves of SCB control is smoother than that of PD+NL control.

Remark 4 To estimate the lower bound of $f_{i}$ for stable tracking control of the 2DOF manipulator, we calculate the value of $f_{c 2}$ which equals 4624 . By Theorem 1 , only if $f_{i}>4624$, local convergence of the tracking errors may be ensured. But the simulation results show that the tracking errors are already convergent when $f_{i}<<f_{c 2}$. Theorem 1 only gives conservative sufficient conditions for convergence in quantity, however, its importance is that the existence of bounded $f_{i}$ ensuring the stability and robust properties of the controlled system is proved theoretically as shown in section 4 . 


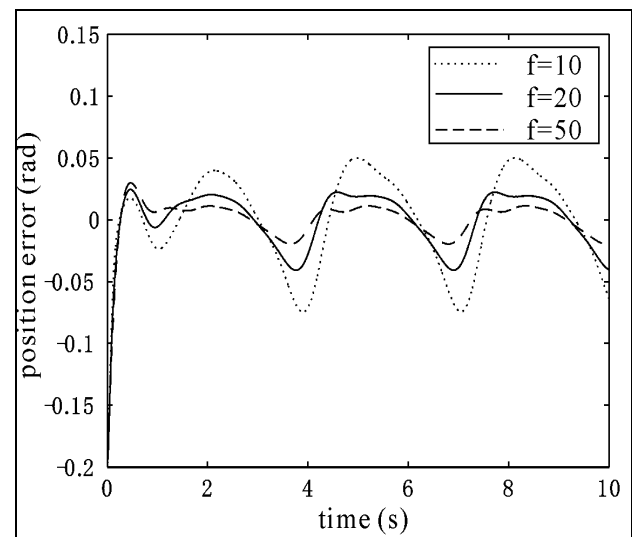

Fig. 2. Position tracking errors of joint 1 for different $f_{i}$ 's values.

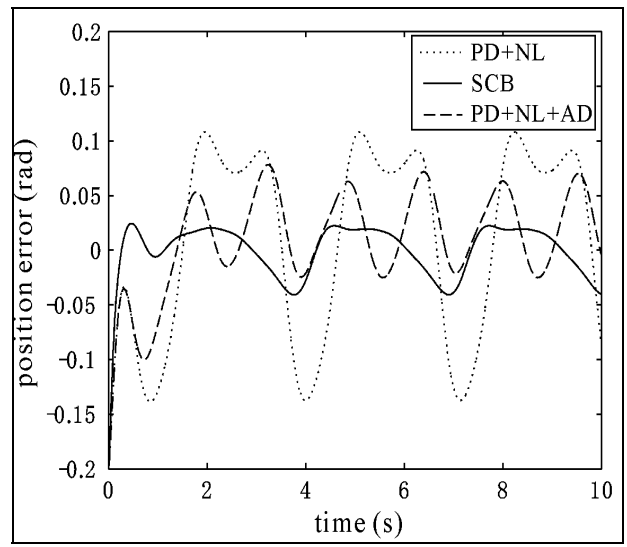

Fig. 4. Position tracking errors of joint 1.

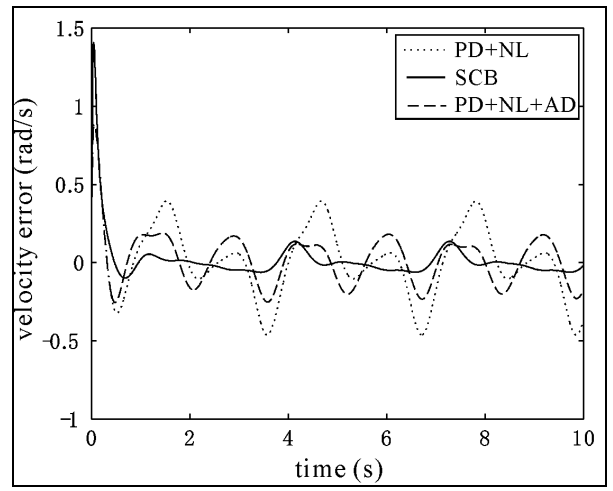

Fig. 6. Velocity tracking errors of joint 1.

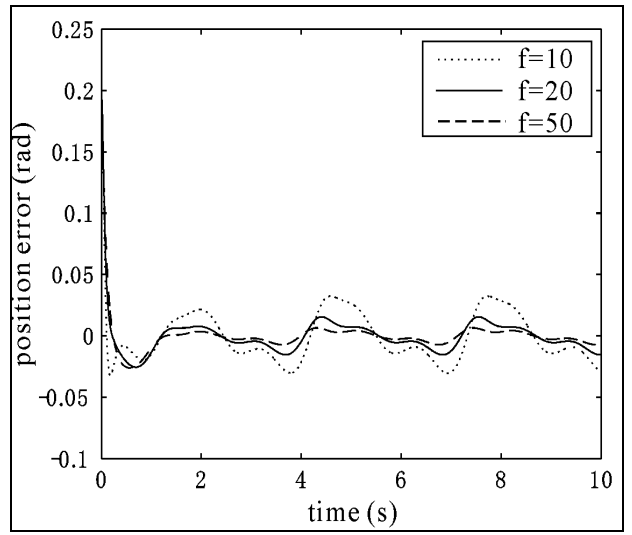

Fig. 3. Position tracking errors of joint 2 for different $f_{i}$ 's values.

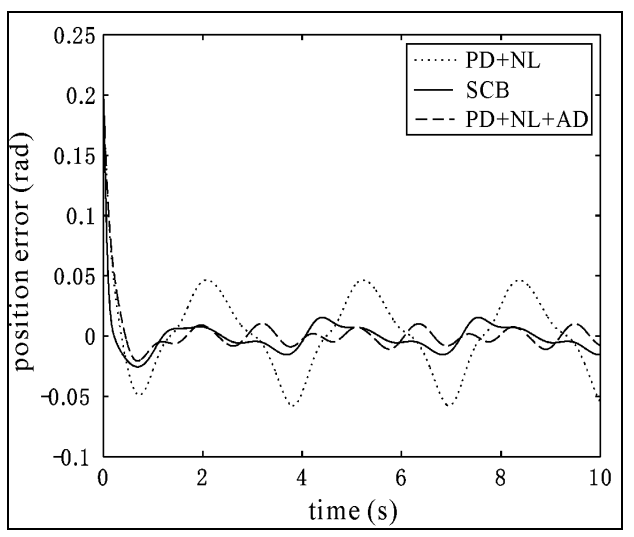

Fig. 5. Position tracking errors of joint 2.

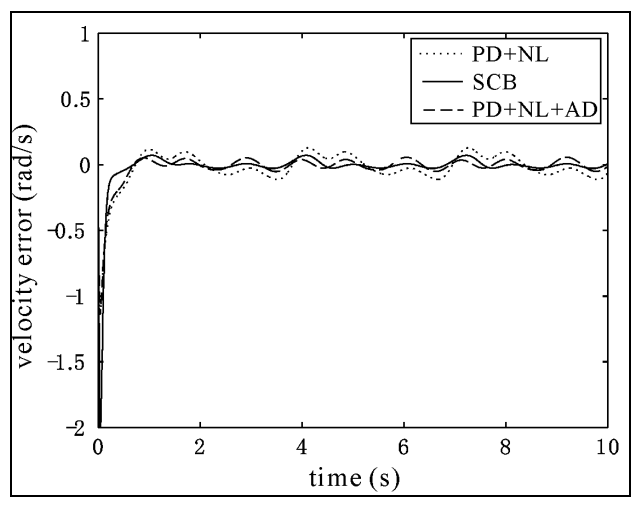

Fig. 7. Velocity tracking errors of joint 2. 


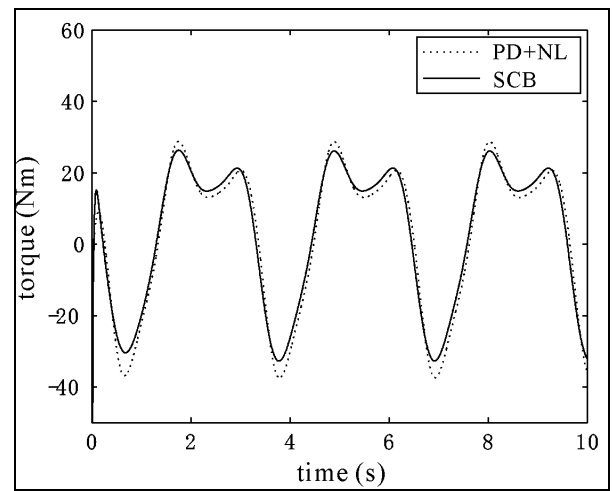

Fig. 8. Input torques of joint 1.

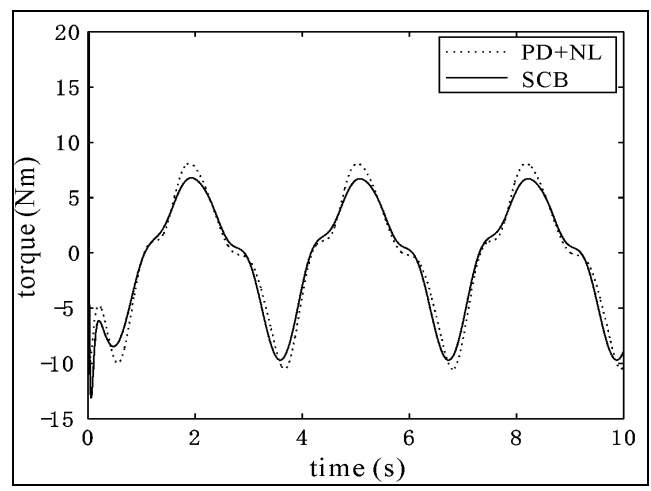

Fig. 9. Input torques of joint 2.

\section{Conclusions}

The controller designed by the method proposed in this chapter consists of a feedforward and a two-loop linear time-invariant robust controller. Because the robust compensation signal is generated based on the inner signals, there is no need to know the format of uncertainties. In addition, only using local joint position feedbacks, the noise problem possibly caused by velocity measurement is avoided. The most important is that the parameter tuning is monotonic which provides the unique superiority: tuning on-line is realizable, and the bound estimation of uncertainties is not needed. These characteristics enable the controller possess favorable adaptability and be prone to be realized easily.

\section{References}

Canudas De Wit C.; Siciliano, B. \& Bastin, G. (Ed.), (1996). Theory of Robot Control, 78-103, Springer, London

$\mathrm{Fu}$, L. (1992). Robust adaptive decentralized control of robot manipulators. IEEE Trans. Automatic Control, Vol.37, 106-110

Kelly, R. \& Salgado, R. (1994). PD control with computed feedforward of robot manipulators: a design procedure. IEEE Trans. Robotics and Automation, Vol.10, 566-571

Kelly, R. (1997). PD control with desired gravity compensation of robotic manipulators: A review. Int. J. robotics research, Vol.16, 660-672

Liu, G. \& Goldenberg, A. (1996). Uncertainty decomposition -based robust control of robot manipulators. IEEE Trans. Control Systems Technology, Vol.4, 384-393

Liu, G. \& Goldenberg, A. (1997). Robust control of robot manipulators based on dynamics decomposition. IEEE Trans. Robotics and Automation, Vol.13, 783-789

Liu, M. (1997). Decentralized PD and robust nonlinear control for robot manipulators. J. Intelligent and Robotic Systems: Theory \& Applications, Vol.20, 319-332

Liu, M. (1999). Decentralized control of robot manipulators: nonlinear and adaptive approaches. IEEE Trans. Automatic Control, Vol.44, 357-363

Qu, Z. ; Dawson, D. \& Lim, S. (1994). A new class of robust control laws for tracking of robots. Int. J. Robotics Research, Vol.13, 355- 363

Sage, H. \& De Mathelin M. (1999). Robust control of robot manipulators: a survey. Int. J. Control, Vol. 72, 1498-1522 
Slotine, J. \& Li, W. (1987). On the adaptive control of robot manipulators. Int. J. Robotics Research, Vol.6, 49-59

Spong, M. \& Thorp, J. (1987). Robust microprocessor control of robot manipulators. Automatica, Vol. 23, 373-379

Spong, M. (1992). On the robust control of robot manipulator. IEEE Trans. Automatic Control, Vol. 37, 1782-1786

Tang, Y. \& Guerrero, G. (1998). Decentralized robust control of robot manipulators. ACC'98, pp.922-926

Wang, J. \& Wend, H. (1999). Robust decentralized control of robot manipulators. Int. J. Systems Science, Vol.30, 323-330

Zhong, Y. ; Nagai, Y. \& Takeuchi, Y. (1999). Robust output tracking of a class of nonlinear time-varying uncertain systems. Proceedings of the 14th IFAC World Congress, vol. G, pp.425-430, Beijing

Zhong, Y. (2002). Robust output tracking control of SISO plants with multiple operating points and with parametric and unconstructed uncertainties. Int. J. Control, Vol.75, 219- 241 


\section{Appendix: Proofs of Lemma $1 \sim$ Lemma 3}

\section{Proof of Lemma 1:}

Solving (17), we can find that $\boldsymbol{\psi}_{i}=\boldsymbol{P}_{i} \boldsymbol{B}_{q i}$ can be expressed in the form as stated in the lemma, and under the condition that $1 / p_{i} \gg>\geq 1, \gamma_{j i}$ can be represented as

$$
\begin{gathered}
\gamma_{j i}=\frac{a_{2 i} f_{i}^{2}+a_{1 i} f_{i}+a_{0 i}}{f_{i}^{2}+\left(\alpha_{1 i}+\beta_{1 i}\right) f_{i}+\left(\alpha_{1 i} \beta_{1 i}+\beta_{2 i}\right)+\left(\alpha_{1 i} \beta_{2 i}\right) f_{i}^{-1}}, j=1,2,3 \\
\gamma_{j i}=\frac{b_{3 i} f_{i}^{3}+b_{2 i} f_{i}^{2}+b_{1 i} f_{i}+b_{0 i}}{f_{i}^{3}+\left(\alpha_{1 i}+\beta_{1 i}\right) f_{i}^{2}+\left(\alpha_{1 i} \beta_{1 i}+\beta_{2 i}\right) f_{i}+\alpha_{1 i} \beta_{2 i}}, j=4,5
\end{gathered}
$$

where $a_{j i}(j=0,1,2)$ and $b_{k i}(k=0,1,2,3)$ are constants independent of $f_{i}$. Since $\alpha_{1 i}, \beta_{1 i}, \beta_{2 i}>0$, obviously, the denominator of $\gamma_{j i}$, denoted by $\operatorname{den}\left(\gamma_{j i}\right)$, is larger than zero, so $\gamma_{j i}$ is continuous in $f_{i}$ and is bounded by constants which are independent of $f_{i}$.

\section{Proof of Lemma 2:}

From (5), we have

$$
\begin{aligned}
\|\boldsymbol{\Delta}\| \leq & \left\|\boldsymbol{M}(\boldsymbol{\theta})-\boldsymbol{M}\left(\boldsymbol{\theta}_{d}\right)\right\|\left\|\ddot{\boldsymbol{\theta}}_{d}\right\|+\left\|\boldsymbol{M}\left(\boldsymbol{\theta}_{d}\right) \ddot{\boldsymbol{\theta}}_{d}-\boldsymbol{M}_{0}\left(\boldsymbol{\theta}_{d}\right) \ddot{\boldsymbol{\theta}}_{d}\right\|+\left\|\boldsymbol{C}(\boldsymbol{\theta}, \dot{\boldsymbol{\theta}})-\boldsymbol{C}\left(\boldsymbol{\theta}_{d}, \dot{\boldsymbol{\theta}}_{d}\right)\right\|\left\|\dot{\boldsymbol{\theta}}_{d}\right\| \\
& +\left\|\boldsymbol{C}\left(\boldsymbol{\theta}_{d}, \dot{\boldsymbol{\theta}}_{d}\right) \dot{\boldsymbol{\theta}}_{d}-\boldsymbol{C}_{0}\left(\boldsymbol{\theta}_{d}, \dot{\boldsymbol{\theta}}_{d}\right) \dot{\boldsymbol{\theta}}_{d}\right\|+\left\|\boldsymbol{g}(\boldsymbol{\theta})-\boldsymbol{g}\left(\boldsymbol{\theta}_{d}\right)\right\|+\left\|\boldsymbol{g}\left(\boldsymbol{\theta}_{d}\right)-\boldsymbol{g}_{0}\left(\boldsymbol{\theta}_{d}\right)\right\|
\end{aligned}
$$

Suppose the desired trajectory is bounded and satisfies

$$
\left\|\boldsymbol{\theta}_{d}\right\| \leq c_{1},\left\|\dot{\boldsymbol{\theta}}_{d}\right\| \leq c_{2},\left\|\ddot{\boldsymbol{\theta}}_{d}\right\| \leq c_{3}
$$

where $c_{1}, c_{2}$ and $c_{3}$ are constants. In the view of Property 1 3 stated in Section2, there exist positive constants $k_{M}, k_{M 1}, k_{C}, k_{C 1}, k_{C 2}$, satisfying

$$
\|\boldsymbol{\Delta}\| \leq k_{M} c_{3}+k_{M 1} c_{3}\|\boldsymbol{e}\|+k_{C} c_{2}+k_{C 1} c_{2}\|\dot{\boldsymbol{e}}\|+k_{C 2} c_{2}^{2}\|\boldsymbol{e}\|+k_{g}+k_{g 1}\|\boldsymbol{e}\|
$$

From Property 1 and Property 3(3), there exist positive constants $k_{C 3}, k_{M 2}$ such that

So we have

$$
\left\|-\boldsymbol{M}^{-1} \boldsymbol{C} \dot{\boldsymbol{e}}+\left(\boldsymbol{M}^{-1}-b_{0} \boldsymbol{I}\right) \boldsymbol{u}_{0}\right\| \leq k_{C 3}\|\dot{\boldsymbol{e}}\|+k_{C 4}\|\dot{\boldsymbol{e}}\|^{2}+k_{M 2}\left\|\boldsymbol{u}_{0}\right\|
$$

$$
\|\tilde{\boldsymbol{q}}\| \leq k_{q 1}+k_{q 2}\|\boldsymbol{e}\|+k_{q 3}\|\dot{\boldsymbol{e}}\|+k_{q 4}\|\dot{\boldsymbol{e}}\|^{2}+k_{q 5}\left\|\boldsymbol{u}_{0}\right\|^{2}
$$

where $k_{q 1}, k_{q 2}, \cdots, k_{q 5}$ are bounded positive constants.

\section{Proof of Lemma 3:}

Since $b_{0} \geq \lambda_{\min }\left(\boldsymbol{M}^{-1}\right)$ which implies that $\bar{\sigma}\left(b_{0}^{-1} \boldsymbol{M}^{-1}\right) \leq 1$, so we have

$$
\bar{\delta}\left[b_{0}^{-1} \boldsymbol{M}^{-1}-\boldsymbol{I}\right] \leq 1-b_{0}^{-1} \lambda_{\min }\left(\boldsymbol{M}^{-1}\right)
$$

From Assumption C), it follows that

$$
\bar{\delta}\left[b_{0}^{-1} \boldsymbol{M}^{-1}-\boldsymbol{I}\right] \leq 1-\varepsilon_{\Delta}
$$

Therefore the conclusion holds. 


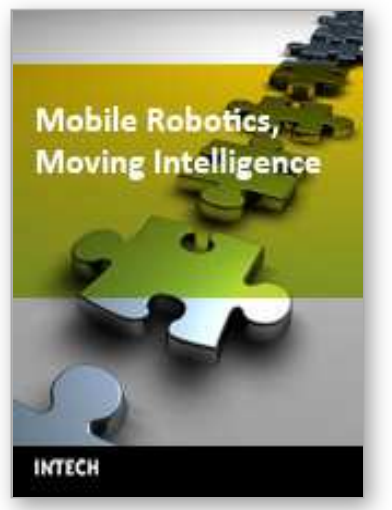

\author{
Mobile Robotics, Moving Intelligence \\ Edited by Jonas Buchli
}

ISBN 3-86611-284-X

Hard cover, 586 pages

Publisher Pro Literatur Verlag, Germany / ARS, Austria

Published online 01, December, 2006

Published in print edition December, 2006

This book covers many aspects of the exciting research in mobile robotics. It deals with different aspects of the control problem, especially also under uncertainty and faults. Mechanical design issues are discussed along with new sensor and actuator concepts. Games like soccer are a good example which comprise many of the aforementioned challenges in a single comprehensive and in the same time entertaining framework. Thus, the book comprises contributions dealing with aspects of the Robotcup competition. The reader will get a feel how the problems cover virtually all engineering disciplines ranging from theoretical research to very application specific work. In addition interesting problems for physics and mathematics arises out of such research. We hope this book will be an inspiring source of knowledge and ideas, stimulating further research in this exciting field. The promises and possible benefits of such efforts are manifold, they range from new transportation systems, intelligent cars to flexible assistants in factories and construction sites, over service robot which assist and support us in daily live, all the way to the possibility for efficient help for impaired and advances in prosthetics.

\title{
How to reference
}

In order to correctly reference this scholarly work, feel free to copy and paste the following:

Zongying Shi, Yisheng Zhong and Wenli Xu (2006). Decentralized Robust Tracking Control for Uncertain Robots, Mobile Robotics, Moving Intelligence, Jonas Buchli (Ed.), ISBN: 3-86611-284-X, InTech, Available from:

http://www.intechopen.com/books/mobile_robotics_moving_intelligence/decentralized_robust_tracking_control _for_uncertain_robots

\section{INTECH}

open science | open minds

\section{InTech Europe}

University Campus STeP Ri

Slavka Krautzeka 83/A

51000 Rijeka, Croatia

Phone: +385 (51) 770447

Fax: +385 (51) 686166

www.intechopen.com

\section{InTech China}

Unit 405, Office Block, Hotel Equatorial Shanghai

No.65, Yan An Road (West), Shanghai, 200040, China

中国上海市延安西路65号上海国际贵都大饭店办公楼405单元

Phone: +86-21-62489820

Fax: +86-21-62489821 
(C) 2006 The Author(s). Licensee IntechOpen. This chapter is distributed under the terms of the Creative Commons Attribution-NonCommercialShareAlike-3.0 License, which permits use, distribution and reproduction for non-commercial purposes, provided the original is properly cited and derivative works building on this content are distributed under the same license. 